\title{
Evaluation of the Knowledge and Perceptions with Regards to Pictorial Health Warnings on Tobacco Products among Tobacco Users Diagnosed with Head and Neck Carcinoma: a Study from the Kumaon Hills of India
}

\author{
Nirdosh Kumar Pant ${ }^{1}$, Kailash Chandra Pandey ${ }^{1}$, Irappa Madabhavi ${ }^{2}$, Vinod \\ Pandey $^{1 *}$, Swaroop Revannasiddaiah ${ }^{1}$
}

\begin{abstract}
Background: Tobacco products continue to be used in large quantities in India despite the mandatory inclusion of pictorial health warnings (PHWs) on all tobacco packaging. The circumstances as to how people could continue the use of tobacco to the point of developing head and neck cancer despite enhanced awareness about the ill effects of tobacco is the main focus of this study. Materials and Methods: This study concerned patients with least 5-years history of tobacco use, having been diagnosed with histopathologically proven malignancies of the hypopharynx, larynx, oropharynx and oral cavity presenting at the Government Medical College-Haldwani, Nainital, India. A total of 183 patients were eligible for inclusion during July 1 2013- June 302014 . Of these, 59 patients used smoked tobacco exclusively, 22 patients used smokeless tobacco exclusively, and 102 patients used both forms of tobacco. Among users of smoked forms, $75.2 \%(n=121)$ were beedi users, and 24.8\% (n=40) were cigarette users. Patients were asked direct questions as to whether they had noticed the presence of PHWs upon tobacco products. The reasons as to why PHWs were not effective in stopping the patients from tobacco use were investigated. Results: Of the 183 patients, 146 reported being aware about the presence of PHWs, and when they were asked reasons as to why they continued tobacco despite being aware of ill-effects, the commonest reason chosen (by 53.4\%) was that patients had not regarded themselves as using tobacco heavy enough to cause cancer. Among the 36 patients who reported as being oblivious to the presence of PHWs on tobacco products, 63.9\% reported that the products they used never displayed any PHWs, and $36.1 \%$ reported never having paid attention to the packaging. The awareness about PHWs was higher among cigarette smokers in comparison to beedi smokers $(\mathbf{1 0 0 \%}$ vs $\mathbf{7 6 . 1 \%}, \mathbf{p = 0 . 0 0 0 2})$. Conclusions: Locally produced and marketed tobacco products such as beedis and oral tobacco often fail to display PHWs. The presence of PHWs without doubt enhances awareness about the carcinogenic risks of tobacco. However, enhanced awareness alone may not be enough, and as elucidated by this study, some persons continue to use tobacco to the point of developing malignancies. The need of the hour is the implementation of legal and economic sanctions discouraging the use of tobacco products.
\end{abstract}

Keywords: Pictorial health warnings - health warning labels - smoking health warning - head and neck cancer

Asian Pac J Cancer Prev, 15 (18), 7891-7895

\section{Introduction}

The use of tobacco is a major cause of mortality and morbidity in this era. Tobacco use is widespread and ubiquitous in the world, with it being used both in smoked forms (cigarettes, beedis, etc) and non-smoked forms (guthka, pan-masala etc). Tobacco users have a proven higher risk of malignancies, as well as non-malignant serious disorders such as cardiovascular and pulmonary disorders (Zarocostas, 2011; Bhawna, 2013).

In an attempt to reduce the magnitude of tobacco use, various nations have adopted compulsory inclusion of pictorial health warnings (PHWs) upon all tobacco products. The same have been implemented in India, and since 2009 , have been mandated by law as compulsory by the Cigarettes and other tobacco products act (COTPA) (Tripathy et al., 2013). Warning pictures on tobacco products have two primary intentions - to encourage current tobacco users to quit using, and to discourage non-users from initiating tobacco use (Cantrell et al., 2013; Volchan et al., 2013).

Despite the implementation of pictorial warnings, there happens to be a persistent high prevalence of tobacco use. This study was one among the series of tobacco related

${ }^{1}$ Department of Radiotherapy, Government Medical College-Haldwani, Haldwani, ${ }^{2}$ Department of Medical Oncology, Gujarat Cancer Research Institute ,Ahmedabad, India *For correspondence: swarooptheone@ gmail.com 
studies conducted at the Swami Rama Cancer Hospital and Research institute during 2013-14. The hospital runs as part of the Government Medical College-Haldwani, Nainital and serves the patients from the Kumaon Hills of India. This study was designed with the intention to assess the perceptions and knowledge with regards to the pictorial health warnings in a specific population- that is a population of tobacco-users who are diagnosed with squamous cell carcinoma of the head and neck (SCCHN).

This study was conducted to assess the perceptions and knowledge with regards to the pictorial health warnings upon tobacco products in a specific population confined to tobacco-user patients diagnosed with squamous cell carcinoma of the head and neck (SCCHN). We expected to learn the impact of PHWs upon the perceptions regarding tobacco use and also the reasons as to how and why the patients continued tobacco use to the point of developing malignancy.

\section{Materials and Methods}

The study design employed was observational and cross-sectional in approach, in that the analysis was confined to a specific population, i.e. of patients diagnosed with SCCHN and having had a positive history of chronic tobacco use. For the time span between 1 July 2013- 30 June 2014, a total of 206 patients of SCCHN (with a positive history of tobacco use for more than 5-years) were registered at the Department of Radiotherapy, Swami Rama Cancer Hospital and Research Institute, Haldwani, Nainital, in India. After excluding patients with carcinoma of the nasopharynx, maxillary antrum, nasal cavity and also those patients with secondary in a neck node with unknown primary, a total of 183 patients of SCCHN were included for the study.

Of these 183 patients, male patients numbered 167 $(91.3 \%)$ and female patients numbered $16(8.7 \%)$. With regards to the subsite of SCCHN, the number of patients with carcinoma of the oral cavity, larynx, oropharynx and hypopharynx were $71(38.8 \%), 49(26.8 \%), 41(22.4 \%)$ and $22(12.1 \%)$, respectively. With regards to the form of tobacco use- 59 patients responded as being exclusive users of smoked tobacco, 22 patients responded as being exclusive users of smokeless tobacco, while 102 patients responded as being users of both smoked and smokeless forms of tobacco. Among the 161 users of smoked forms of tobacco, $121(75.2 \%)$ responded as being beedi users, while $40(24.8 \%)$ were cigarette users.

All the patients were asked a direct question as to whether they had noticed the presence of pictorial health warnings (PHW) over the tobacco products they have been using. Patients responding as having had noticed PHWs were further asked questions listed in 'questionnaire-A' (Table 1). Patients who responded as not having knowledge about the PHWs were asked questions listed in 'questionnaire-B' (Table 2).

\section{Results}

Of the 183 patients, $146(79.8 \%)$ reported as being aware about the presence of PHWs, while 36 (20.2\%) reported as being oblivious to the presence of PHWs on tobacco products. Among patients reporting as having had noticed the presence of PHWs, 137 did report that PHWs were effective at educating people about the carcinogenic ability of tobacco products (Figure 1). Despite being aware of the ill effects of tobacco, patients were asked as to how they could continue tobacco use to the point of developing head and neck cancer. Majority (53.4\%) reported as not having regarded themselves as using tobacco in quantities enough to cause cancer. A reason chosen by 15 patients $(10.3 \%)$ was that they initiated use of tobacco before PHWs were being displayed upon the products. Also, nine patients $(6.2 \%)$ did confess that they had not perceived the warnings seriously (Figure 2).

Majority of the respondents $(56.2 \% ; n=82)$ who did declare as having been aware of PHWs were of the opinion that they were unsure as to whether PHWs will be useful in reducing burden of tobacco induced cancers in the society. Only 28 patients $(19.2 \%)$ felt that PHWs had the potential to reduce burden of tobacco induced cancers, and $36(24.7 \%)$ of these patients were of the opinion that PHWs had no potential to reduce burden of tobacco induced cancers (Figure 3).

Among the 36 patients who declared as not having knowledge about PHWs, majority $(n=29,80.6 \%)$ were beedi users and the remaining were oral tobacco users $(n=7,19.4 \%)$. It was noteworthy that none among the 36

Table 1. Questionnaire Asked for Patients Responding as Having Noticed the Presence of PHWs Upon Tobacco Products they have Been using

1. Were the pictorial warnings effectively educative of the carcinogenic ability of tobacco products in a serious manner?

Yes

No

2. Why did you continue using tobacco despite being made aware of the ill effects via the pictorial warnings? Choose the most appropriate answer Did not perceive the warnings seriously

Did not regard myself as a heavy tobacco user to get cancer

Had already used tobacco for years before PHWs began appearing Did try to quit, but had not been successful

3. Do you think pictorial warnings will be useful in reducing burden of tobacco induced cancers in the society to any measurable extent?

Yes

No

May be/ Don't know

Table 2. Questionnaire Asked for Patients Responding as Having no Knowledge about PHWs

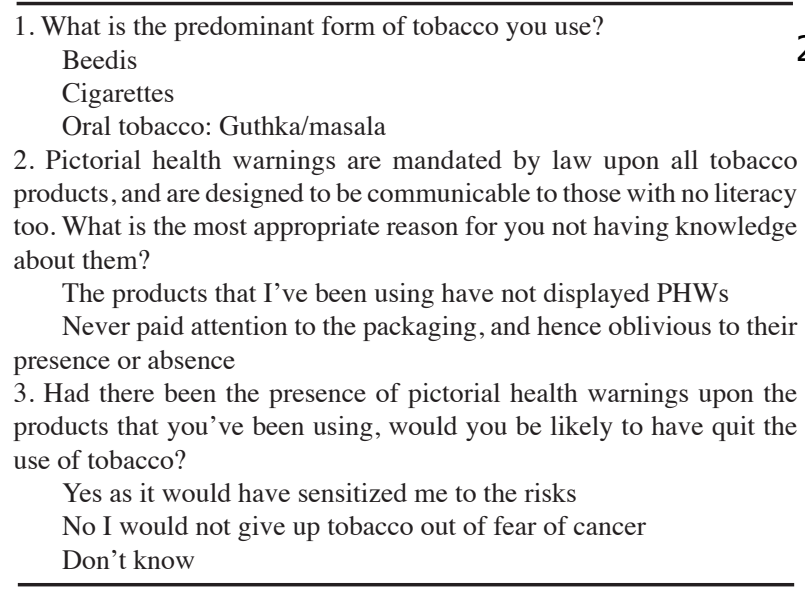


patients were cigarette smokers (Figure 4). Among those 36 patients, $63.9 \%(n=23)$ reported that their products did not contain PHWs, while $36.1 \%(n=13)$ reported that they never paid attention to packaging and were hence oblivious to their presence or absence upon the tobacco products (Figure 5).

When patients who reported not having seen PHW asked as to whether they would have quit the use of tobacco in case they had seen PHWs, 66.7\% $(n=24)$ responded that they would not have given up tobacco just out of the fear of cancer.

Among the 161 users of smoked forms of tobacco, 121 (75.2\%) responded as being beedi users, while $40(24.8 \%)$ responded as being cigarette users. The awareness about PHWs was higher among cigarette smokers in comparison to beedi smokers. While $100 \%$ of cigarette smokers $(n=40$ of 40) were aware about PHWs, only 76.1\% ( $\mathrm{n}=92$ of 121). This difference was statistically significant by the Fisher Exact Test $(\mathrm{p}=0.0002)$.

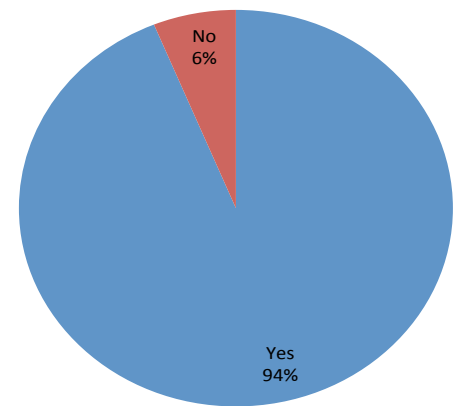

Figure 1. Opinion Regarding Whether Pictorial Warnings are Effectively Educative of the Carcinogenic Ability of Tobacco Products in a Serious Manner

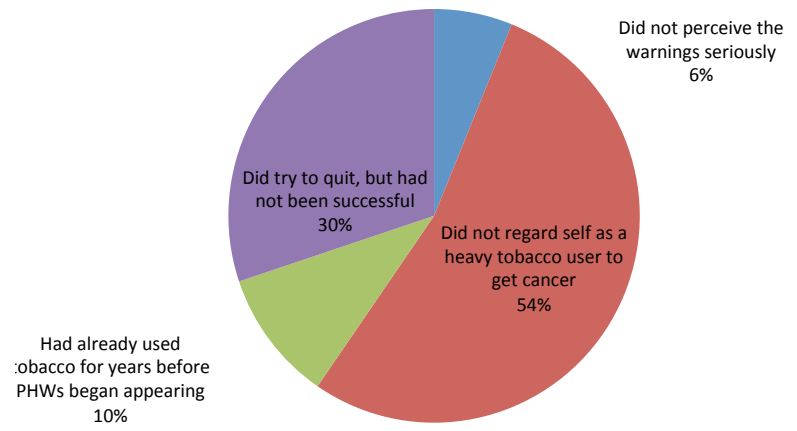

Figure 2. Reason for Contining using Tobacco Despite Being Made Aware of the Ill Effects Via the Pictorial Warnings

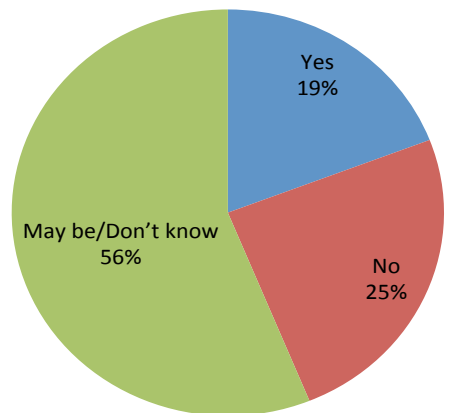

Figure 3. Opinion about Whether Pictorial Warnings will be Useful in Reducing Burden of Tobacco Induced Cancers in the Society?
DOI:http://dx.doi.org/10.7314/APJCP.2014.15.18.7891 Pictorial Health Warnings on Tobacco Products in India.

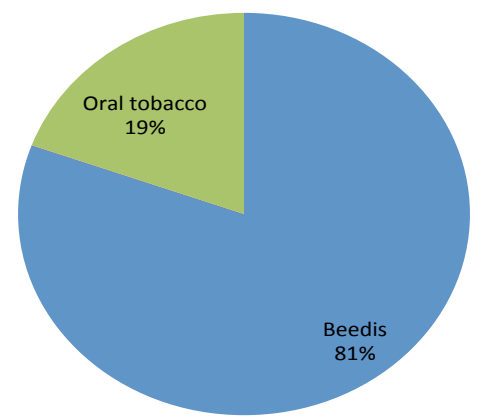

Figure 4. Predominant form of Tobacco use Among those with no Knowledge of PHWs?

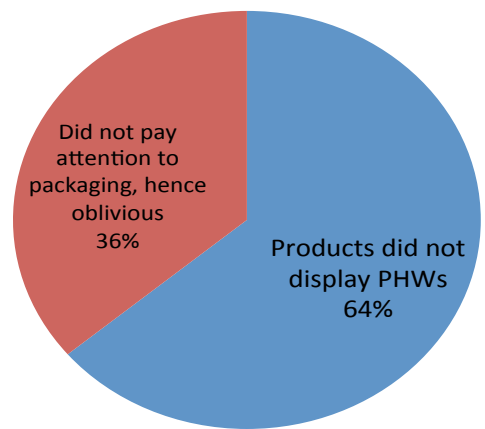

Figure 5. The Most Appropriate Reason for not having Knowledge about PHWs?

\section{Discussion}

Tobacco products are among the commonest form of substance abuse worldwide. Owing to the presence of the psychoactive substance nicotine, addiction ensures as a result of psychological and physical dependence. The problem of worldwide tobacco use is rather colossal, with an estimate of over a billion active tobacco users worldwide. About 1/3rd of the world adult population uses tobacco, and in India, 30\% of the populations above 15 years of age are tobacco users (Rani et al., 2003; Balagopal et al., 2012).

The world health organization (WHO) estimates that tobacco causes 5.4 million deaths per year worldwide, and hence, is considered as the single most significant preventable cause of mortality and morbidity worldwide (WHO report, 2008). Tobacco is not only associated with malignancies, but also with serious non-cancerous illnesses which are life-threatening and life-limiting in their own rights. The causal and contributory association of tobacco towards serious conditions such as coronary heart disease, atherosclerosis, peripheral vascular diseases, chronic bronchitis, emphysema, etc is established beyond doubt (Apslund, 2003; Henley et al., 2004; Sharma et al., 2011; Talikka et al., 2012; Al-Attas et al., 2014).

Given the alarming risks associated with tobacco owing to direct health implications upon individuals' family and society at large, there have been various efforts by governmental and non-governmental agencies so as to reduce, or discourage the use of tobacco. It was, and continues to be often assumed that lack of awareness about the ill-effects of tobacco is an important cause behind the extensive prevalence of tobacco use in the society. One of the most visible, and presumably a very effective means of enhancing awareness about the ill effects of tobacco 
has been the inclusion of PHWs upon tobacco products' packaging. In fact, PHWs are one of the six MPOWER strategies devised by the WHO in order to combat tobacco use (Kaleta et al., 2009; Spires et al., 2014).

The WHO adopted the Framework Convention on Tobacco Control (FCTC) in the year 2003, and this provides a set of guidelines for controlling the demand and supply of tobacco. The FCTC states that "every person should be informed of the health consequences, addictive nature and mortal threat posed by tobacco consumption and exposure to tobacco smoke" (WHO, 2003; Collishaw, 2010; Ullrich et al., 2014). In India, the FCTC was accepted and ratified in 2004. In India, earlier, the Cigarettes and other tobacco products - Prohibition of advertisement and regulation of trade and commerce, production, supply and distribution act COTPA) was formulated by the ministry of law and justice in 2003, and came into force from May 12004 . However, undue delays ensued and PHWs upon tobacco products' packaging were mandated that from 1 June 2009 onwards, all tobacco products which are being retailed in India, including those being imported, have to display PHWs on their packages (Hammond et al., 2006; Aruna et al., 2010).

PHWs serve as a vivid and memorable way to educate users, and potential users about the devastating potential of tobacco. They are by their pictorial design able to communicate irrespective of language barrier or irrespective of the educational status of the intended audience (Süssenbach et al., 2013). The effectiveness of the PHWs increases with factors such as size of the PHW, percentage of the packaging displaying the PHW, position of the PHW upon the packaging, and the color of the PHW. Given the importance of the PHW size, various countries have made it mandatory that at least a pre-defined percentage of the packaging must be covered by the PHW (Bansal-Travers et al., 2011; Mays et al., 2014). In Australia, tobacco products' packaging must contain PHW which covers $90 \%$ of the front, and $30 \%$ of the reverse side of the packaging. Canada and Thailand have made it mandatory that $50 \%$ of the front and reverse side of the packaging must display PHW. Brazil as a matter of fact has made it mandatory that $100 \%$ of the front, as well as the reverse side of the packaging must be covered by the PHW (Cunningham, 2009; Fong et al., 2009; Gigliotti et al., 2014).

The effectiveness of the PHWs in terms of making smokers feel smoking as 'less glamorous and attractive' has been proven in many studies. In a study from Canada, $90 \%$ of the participants agreed that PHWs were effective means of making smokers feel smoking as a less attractive habit (Hitchman et al., 2011). This was reflected in the results of our study too, where in $94 \%$ of cancer patients did report that PHWs were effective in educating about the ill-effects of tobacco.

However, there are two main reasons as to why tobacco products continue to be used despite all above legislations. Firstly, there are many locally produced and marketed items, such as beedis often operate in ignorance or defiance of the requirements of PHWs. Cigarette users were more likely to be aware of the presence of PHWs in comparison to those using beedis. This is likely due to the fact that cigarettes are produced by licensed factories and are regulated by law. Beedis on the other hand are more likely to be prepared in small scale/home based industries which may act outside of the legal requirements (Yen et al., 2000; Sharma et al., 2013).

The other major reason is that mere enhancement of awareness about risks with tobacco use will not be enough to deter patients with strong addictions to quit using tobacco. This is possible because of ego defense mechanisms within the psychological makeup of the patients. Ego defense mechanisms are psychological reactions within the thought flow process that people use to subconsciously avoid experiencing the reality of their situations. They serve the purpose of maintaining a selfimage despite obvious cause for emotional/psychological distress. Though defense mechanisms serve to help individuals to cope up with routine situations they are pathological if they are used to ignore the warning signs of something known to be harmful, or wrong (Bell, 1965; Abrams, 1968; Kessels et al., 2014).

There are various defense mechanisms describednamely rationalization, intellectualization, minimization, denial, suppression, hostility, avoidance, etc. These are known to operate outside of the conscious awareness of a person (Sherman et al., 2000; Wilson, 2002). The above constitutes what could be called as a 'psychological immune system', which helps in maintaining a positive self-image despite negative reactions (C Ruiter, 2005).

Thus, these defense mechanisms serve mainly to get rid of fear, and not of the threat. In presence of strong defense mechanisms, mere awareness programs such as PHWs cannot be expected to be of major significance. More stringent measures- political, legal, and economic measures must be taken.

In conclusion, PHWs are in general present upon factory manufactured tobacco products, and occasional local made tobacco products do not contain PHWs. PWHs are generally effective in educating tobacco users regarding the associated risks of malignancies. However, PHWs are in themselves not enough to curtail the tobacco use in the society, since tobacco users' psychological defense mechanisms operate to negate the effects of the PHWs. is a dire need for effective anti-tobacco legislations, and for the placement of legal restrictions against the use of, and the sale of tobacco products. Strict laws discouraging the use of tobacco, and imposing heavy taxation upon tobacco products may help reduce the use of tobacco products in the society. It has after-all been observed that (WHO report, 1997) for every $10 \%$ increase in the cost of tobacco will reduce the consumption by $2-8 \%$.

\section{References}

Abrams A, Gagnon JH, Levin JJ (1968). Psychosocial aspects of addiction. Am J Public Health Nations Health, 58, 2142-55.

Al-Attas SA, Ibrahim SS, Amer HA, et al (2014). Prevalence of potentially malignant oral mucosal lesions among tobacco users in Jeddah, Saudi Arabia. Asian Pac J Cancer Prev, 15, 757-62.

Aruna DS, Rajesh G, Mohanty VR (2010). Insights into pictorial health warnings on tobacco product packages marketed in 
Uttar Pradesh, India. Asian Pac J Cancer Prev, 11, 539-43. Asplund K (2003). Smokeless tobacco and cardiovascular disease. Prog Cardiovasc Dis, 45, 383-94.

Balagopal PG, George NA, Venugopal A, et al (2012). Tobacco related habits among first degree relatives of patients undergoing surgery for advanced head and neck malignancies in India. Asian Pac J Cancer Prev, 13, 217-20.

Bansal-Travers M, Hammond D, Smith P, Cummings KM (2011). The impact of cigarette pack design, descriptors, and warning labels on risk perception in the U.S. Am J Prev Med, 40, 674-82.

Bell RG (1965). Defensive thinking in alcohol addicts. Can Med Assoc J, 92, 228-31.

Bhawna G (2013). Burden of smoked and smokeless tobacco consumption in India - results from the Global adult Tobacco Survey India (GATS-India)- 2009-2010. Asian Pac J Cancer Prev, 14, 3323-9.

C Ruiter RA, Kok G (2005). Saying is not (always) doing: cigarette warning labels are useless. Eur J Public Health, $15,329$.

Cantrell J, Vallone DM, Thrasher JF, et al (2013). Impact of tobacco-related health warning labels across socioeconomic, race and ethnic groups: results from a randomized web-based experiment. PLoS One, $\mathbf{8}, 52206$.

Collishaw NE (2010). The millennium development goals and tobacco control. Glob Health Promot, 17, 51-9.

Cunningham R (2009). Gruesome photos on cigarette packages reduce tobacco use. Bull WHO, 87, 569 .

Fong GT, Hammond D, Hitchman SC (2009). The impact of pictures on the effectiveness of tobacco warnings. Bull WHO, 87, 640-3.

Gigliotti A, Figueiredo VC, Madruga CS, et al (2014). How smokers may react to cigarette taxes and price increases in Brazil: data from a national survey. BMC Public Health, 14, 327

Hammond D, Fong GT, McNeill A, (2006). Effectiveness of cigarette warning labels in informing smokers about the risks of smoking: findings from the International Tobacco Control (ITC) Four Country Survey. Tob Control, 15, 19-25.

Henley SJ, Thun MJ, Chao A, Calle EE (2004). Association between exclusive pipe smoking and mortality from cancer and other diseases. J Natl Cancer Inst, 96, 853-61.

Hitchman SC, Driezen P, Logel C, et al (2014). Changes in effectiveness of cigarette health warnings over time in Canada and the United States, 2002-2011. Nicotine Tob Res, 16, 536-43.

Kaleta D, Koziel A, Miskiewicz P (2009). MPOWER--strategy for fighting the global tobacco epidemic. Med Pr, 60, 145-9.

Kessels LT, Ruiter RA, Wouters L, Jansma BM (2014). Neuroscientific evidence for defensive avoidance of fear appeals. Int J Psychol, 49, 80-8.

Mays D, Niaura RS, Evans WD, (2014). Cigarette packaging and health warnings: the impact of plain packaging and message framing on young smokers. Tob Control, doi: 10.1136/tobaccocontrol-2013-051234. [Epub ahead of print]

Rani M, Bonu S, Jha P, et al (2003). Tobacco use in India: prevalence and predictors of smoking and chewing in a national cross sectional household survey. Tob Control, 12, 4 .

Sharma MK, Gour N,Pandey A, Wallia D (2011). Epidemiological study of risk factors for oral, laryngeal and esophageal cancers at a tertiary care hospital in India. Asian Pac $J$ Cancer Prev, 12, 1215-8.

Sharma PK, Bansal R (2013). Profile of lung cancer in predominantly Bidi smoking rural population of northern Himachal Pradesh. Indian J Chest Dis Allied Sci, 55, 75-8.

Sherman DAK, Nelson LD, Steele CM (2000). Do messages about health risks threaten the self? Increasing the acceptance
DOI:http://dx.doi.org/10.7314/APJCP.2014.15.18.7891 Pictorial Health Warnings on Tobacco Products in India.

of threatening health messages via self-affirmation. Pers Soc Psychol Bull, 26, 1046-58.

Spires M, Rutkow L, Feldhaus I, Cohen JE (2014). The World Health Organization's MPOWER framework and international human rights treaties: an opportunity to promote global tobacco control. Public Health, 128, 665-7.

Süssenbach P, Niemeier S, Glock S (2013). Effects of and attention to graphic warning labels on cigarette packages. Psychol Health, 28, 1192-206.

Talikka M, Sierro N, Ivanov NV, et al (2012). Genomic impact of cigarette smoke, with application to three smoking-related diseases. Crit Rev Toxicol, 42, 877-89.

Tripathy JP, Goel S, Patro BK (2013). Compliance monitoring of prohibition of smoking (under section-4 of COTPA) at a tertiary health-care institution in a smoke-free city of India. Lung India, 30, 312-5.

Ullrich A, Miller A (2014). Global response to the burden of cancer: the WHO approach. Am Soc Clin Oncol Educ Book. 2014, 311-5.

Volchan E, David IA, Tavares G, et al (2013). Implicit motivational impact of pictorial health warning on cigarette packs. PLoS One, 8, 72117.

WHO Framework Convention on Tobacco Control. Geneva: World Health Organization; 2003. World Health Organization.

Wilson TD (2002). Strangers to ourselves: Discovering the adaptive unconscious. Cambridge, MA: The Belknap Press of Harvard University Press.

World Health Organization (1997). Tobacco or health : a global status report. World Health Organization, Geneva.

World Health Organization (2008). WHO report on the Global Tobacco Epidemic, 2008. Available at HYPERLINK "http:// whqlibdoc.who.int/publications/2008/9789241596282_eng. pdf"

Yen KL, Hechavarria E, Bostwick SB (2000). Bidi cigarettes: An emerging threat to adolescent health. Arch Pediatr Adolesc Med, 154, 1187-9.

Zarocostas J (2011). WHO lists "best buys" for cutting deaths from non-communicable disease. $B M J, \mathbf{3 4 2}, 2648$ 